\title{
A Smashing Subcategory of the Homotopy Category of Gorenstein Projective Modules
}

\author{
Nan Gao
}

Received: 26 October 2012 / Accepted: 8 January 2013 / Published online: 26 July 2013

(C) The Author(s) 2013. This article is published with open access at SpringerLink.com

\begin{abstract}
Let $A$ be an artin algebra of finite CM-type. In this paper, we show that if $A$ is virtually Gorenstein, then the homotopy category of Gorenstein projective $A$-modules, denote $K(A-\mathcal{G P})$, is always compactly generated. Based on this result, it will be proved that the homotopy category of projective $A$-modules, denote $K(A-\mathcal{P})$, is a smashing subcategory of $K(A-\mathcal{G} \mathcal{P})$ and the corresponding Verdier quotient is also compactly generated. Furthermore, it turns out that the inclusion functor $i: K(A-\mathcal{P}) \rightarrow K(A-\mathcal{G P})$ induces a recollement of $K(A-\mathcal{G P})$.
\end{abstract}

Keywords Gorenstein projective modules • Compactly generated homotopy categories $\cdot$ Smashing subcategory $\cdot$ Recollements

\section{Introduction}

Let $\mathcal{X}$ be a class of left modules over an associative ring $R$ which is closed under set-indexed coproducts and direct summands. Holm and Jørgensen [13] study the general question of when the homotopy category $K(\mathcal{X})$ of $\mathcal{X}$ is compactly generated. They give a number of sufficient conditions on $R$ and $\mathcal{X}$ which ensure that $K(\mathcal{X})$ is compactly generated.

Let $A$ be an artin algebra and $A$-Mod the category of $A$-modules. Denote by $A-\mathcal{P}$ the full subcategory of projective $A$-modules, $A-\mathcal{G P}$ the full subcategory of

Supported by the National Natural Science Foundation of China (Grant No. 11101259).

N. Gao $(\varangle)$

Department of Mathematics, Shanghai University,

Shanghai 200444, People's Republic of China

e-mail: gaonanjane@gmail.com 
Gorenstein projective $A$-modules, and $A-\mathcal{G}$ proj the full subcategory of all finitelygenerated Gorenstein projective modules. As is well known, the homotopy category $K(A-\mathcal{P})$ is compactly generated [15, Theorem 2.4$]$.

Gorenstein projective modules and algebras of finite Cohen-Macaulay type receive a lot of attention (See e.g. [1, 4-6, 8-10, 12, 14, 16, 17, 19]). Recall from [4, 6] that an artin algebra $A$ is of finite Cohen-Macaulay type (simply, CM-type) if there are only finitely many isomorphism classes of finitely-generated indecomposable Gorenstein projective $A$-modules. We are interested in the compact generatedness of the homotopy category $K(A-\mathcal{G P})$ of an artin algebra $A$ of finite CM-type.

In Section 2, we first show that if $A$ is virtually Gorenstein of finite CM-type, then $K(A-\mathcal{G P})$ is compactly generated. Next, based on this result, we show that $K(A-\mathcal{P})$ is a smashing subcategory of $K(A-\mathcal{G P})$ and the Verdier quotient $K(A-\mathcal{G P}) / K(A-\mathcal{P})$ is also compactly generated.

The concept of recollement goes back to the work of Beilinson et al. [2]. In Section 3 , we show the existence of recollements of the homotopy category $K(A-\mathcal{G P})$.

\section{Conditions for Compact Generatedness}

Our aim in this section is to show that $K(A-\mathcal{G P})$ is compactly generated provided $A$ is virtually Gorenstein of finite CM-type. So based on the result of Bruns and Herzog [6, Proposition 2.11], and the result of Jørgensen [16], $K(A-\mathcal{P})$ is a smashing subcategory of $K(A-\mathcal{G P})$ and the Verdier quotient $K(A-\mathcal{G} \mathcal{P}) / K(A-\mathcal{P})$ is also compactly generated.

Our strategy for the compact generatedness of $K(A-\mathcal{G P})$ is to give sufficient conditions on $A$. We will use the following lemma.

Lemma 2.1 [4, Theorem 4.10] Let $A$ be an artin algebra. Then $A$ is virtually Gorenstein of finite CM-type if and only if any Gorenstein projective A-module is a direct sum of finitely-generated modules.

Now we are ready to state and prove our first main theorem in this section.

Theorem 2.2 Let $A$ be a virtually Gorenstein artin algebra of finite CM-type. Then $K(A-\mathcal{G P})$ is a compactly generated triangulated category.

Proof Since $A$ is virtually Gorenstein of finite CM-type, we get from Lemma 2.1 that $A-\mathcal{G P}=\operatorname{Add}(A-\mathcal{G}$ proj $)$ which means that $A-\mathcal{G P}$ is contravariantly finite in $A$-Mod, and also each Gorenstein projective module is pure projective which means that every pure exact sequence of modules from $A-\mathcal{G P}$ is split exact. This implies that $K(A-\mathcal{G P})$ is a compactly generated triangulated category by [13, Theorem 3.1].

Recall from [11] that a complex $X^{\bullet}$ is $A$-GP $\mathcal{P}$-acyclic if the induced complex $\operatorname{Hom}_{A}\left(G, X^{\bullet}\right)$ is acyclic for each module in $A-\mathcal{G P}$, and the Gorenstein derived category $D_{g p}(A$-Mod) of an artin algebra $A$ is defined to be the Verdier quotient of the homotopy category $K\left(A\right.$-mod) with respect to the thick subcategory $K_{g p a c}(A$-Mod) which consists of all $A-\mathcal{G} \mathcal{P}$-acyclic complexes. 
Corollary 2.3 Let $A$ be a Gorenstein artin algebra of finite CM-type. Then $D_{g p}(A$-Mod $)$ is compactly generated.

Proof By the assumption on $A$, we see from [3, Corollary 8.3 and Corollary 8.5 ] that $A$ satisfies the conditions on Theorem 2.2. Hence we get that $K(A-\mathcal{G P})$ is a compactly generated triangulated category. By [7, Proposition 3.5] there is a triangleequivalence $D_{g p}(A$-Mod $) \cong K(A-\mathcal{G P})$. This implies that $D_{g p}(A$-Mod $)$ is compactly generated.

For our second main theorem we need a definition and some lemmas.

Recall from [18] that a full subcategory $\mathcal{B}$ of a compactly generated triangulated category $\mathcal{T}$ is smashing if the inclusion $\mathcal{B} \rightarrow \mathcal{T}$ has a right adjoint which preserves coproducts.

Lemma 2.4 [18, Lemma 4.1] Let $\mathcal{B}$ be a smashing subcategory of a compactly generated triangulated category $\mathcal{T}$. Then $\mathcal{T} / \mathcal{B}$ is a compactly generated triangulated category.

Lemma 2.5 [5, Proposition 2.11] Let $\mathcal{T}$ and $\mathcal{T}$ ' be compactly generated triangulated categories, and let $F: \mathcal{T} \rightarrow \mathcal{T}^{\prime}$ be a fully faithful triangle functor which preserves coproducts and compact objects. Then $F$ admits a right adjoint $G: \mathcal{T}^{\prime} \rightarrow \mathcal{T}$ which preserves coproducts.

So in view of the above lemmas, we have the following theorem.

Theorem 2.6 Let $A$ be a virtually Gorenstein artin algebra of finite CM-type. Then $K(A-\mathcal{P})$ is a smashing subcategory of $K(A-\mathcal{G P})$. Moreover, $K(A-\mathcal{G P}) / K(A-\mathcal{P})$ is a compactly generated triangulated category.

Proof By the assumpotion on $A$, we get from Theorem 2.2 that $K(A-\mathcal{G P})$ is compactly generated, and from $[15$, Theorem 2.4$]$ that $K(A-\mathcal{P})$ is compactly generated and each compact object $P^{\bullet}$ is exactly the upper bounded complex of finitelygenerated projective modules. Let $i: K(A-\mathcal{P}) \rightarrow K(A-\mathcal{G P})$ be the inclusion functor. Note that $i$ naturally preserves coproducts. Let $\left\{G_{i}^{\bullet}\right\}_{i \in I}$ be any family objects in $K(A-\mathcal{G P})$. Then we have $\operatorname{Hom}_{K(A-\mathcal{G P})}\left(i P^{\bullet}, \bigsqcup_{i \in I} G_{i}^{\bullet}\right)=\operatorname{Hom}_{K(A-\mathcal{G P})}\left(P^{\bullet}, \bigsqcup_{i \in I} G_{i}^{\bullet}\right) \cong$ $\bigsqcup_{i \in I} \operatorname{Hom}_{K(A-\mathcal{G P})}\left(P^{\bullet}, G_{i}^{\bullet}\right)=\bigsqcup_{i \in I} \operatorname{Hom}_{K(A-\mathcal{G P})}\left(i P^{\bullet}, G_{i}^{\bullet}\right)$. This implies that $i$ preserves compact objects. Hence by Lemma 2.5 we get that $i$ admits a right adjoint $R$ : $K(A-\mathcal{G P}) \rightarrow K(A-\mathcal{P})$ which preserves coproducts. This means $K(A-\mathcal{P})$ is a smashing subcategory of $K(A-\mathcal{G P})$. This implies by Lemma 2.4 that $K(A-\mathcal{G P}) / K(A-\mathcal{P})$ is a compactly generated triangulated category.

\section{Recollements for the Homotopy Category $K(A-\mathcal{G P})$}

In this section, let $A$ be an artin algebra. Based on the compact generatedness of the full subcategory $K(A-\mathcal{P})$ of $K(A-\mathcal{G P})$, we will apply the arguments of Neeman to prove the existence of a recollement of $K(A-\mathcal{G P})$. 
Lemma 3.1 [21, Theorem 4.1], [22, Theorem 8.6.1] Let $F: \mathcal{T} \rightarrow \mathcal{T}$ be a triangle functor between triangulated categories $\mathcal{T}$ and $\mathcal{T}^{\prime}$, where $\mathcal{T}$ is compactly generated.

(1) F admits a right adjoint if and only if it preserves all coproducts.

(2) F admits a left adjoint if and only if it preserves all products.

Theorem 3.2 Let $A$ be an artin algebra. Then the inclusion functor $i: K(A-\mathcal{P}) \rightarrow$ $K(A-\mathcal{G P})$ induces a recollement of the form

$$
K(A-\mathcal{P}) \underset{R}{\stackrel{i}{\leftrightarrows}} K(A-\mathcal{G P}) \underset{\leftrightarrows}{\leftrightarrows} \operatorname{Ker} R
$$

such that $\operatorname{Ker} R \cong K(A-\mathcal{G P}) / K(A-\mathcal{P})$ as triangulated categories.

Proof Since $A$ is an artin algebra, it follows from [15, Theorem 2.4] that $K(A-\mathcal{P})$ is compactly generated. Note that the inclusion functor $i$ naturally preserves all coproducts and products. Then $i$ admits a right adjoint $R$, also a left adjoint. Hence by [20, Theorem 2.2] we have a recollement of the form

$$
K(A-\mathcal{P}) \underset{R}{\stackrel{i}{\leftrightarrows}} K(A-\mathcal{G P}) \underset{\leftrightarrows}{\leftrightarrows} \operatorname{Ker} R
$$

such that $\operatorname{Ker} R \cong K(A-\mathcal{G P}) / K(A-\mathcal{P})$ as triangulated categories.

So in view of the above theorem, we have the following result. Let us begin by recalling some definitions.

Let $\mathcal{T}$ be a triangulated category with the suspension functor $\Sigma$. Recall from [5, Section 2] that a torsion pair in $\mathcal{T}$ is a pair of strict full subcategories $(\mathcal{X} . \mathcal{Y})$ of $\mathcal{T}$ satisfying the following conditions: (1) $\mathcal{T}(\mathcal{X}, \mathcal{Y})=0$; (2) $\Sigma(\mathcal{X}) \subseteq \mathcal{X}$ and $\Sigma^{-1}(\mathcal{Y}) \subseteq$ $\mathcal{Y}$; (3) For any $T \in \mathcal{T}$ there exists a triangle $X_{T} \stackrel{f_{T}}{\rightarrow} T \stackrel{g^{T}}{\rightarrow} Y^{T} \stackrel{h^{T}}{\rightarrow} \Sigma\left(X_{T}\right)$. Then $\mathcal{X}$ is called a torsion class and $\mathcal{Y}$ is called a torsion-free class. A torsion, torsion-free triple, TTF-triple for short, in $\mathcal{T}$ is a triple $(\mathcal{X}, \mathcal{Y}, \mathcal{Z})$ of full subcategories of $\mathcal{T}$ such that the pairs $(\mathcal{X}, \mathcal{Y})$ and $(\mathcal{Y}, \mathcal{Z})$ are torsion pairs.

Now we give a TTF-triple in $K(A-\mathcal{G P})$.

Corollary 3.3 Let $A$ be an artin algebra. Then there exists a TTF-triple $(K(A-\mathcal{P})$, $\left.\operatorname{Ker} R,(\operatorname{Ker} R)^{\perp}\right)$ in $K(A-\mathcal{G P})$.

Proof By Theorem 3.2 we have the recollement of the form

$$
K(A-\mathcal{P}) \underset{R}{\stackrel{i}{\rightleftarrows}} K(A-\mathcal{G P}) \underset{\leftrightarrows}{\leftrightarrows} \operatorname{Ker} R
$$

Hence by [20, Theorem 2.2] we get that $(K(A-\mathcal{P}), \operatorname{Ker} R)$ and $\left(\operatorname{Ker} R,(\operatorname{Ker} R)^{\perp}\right)$ are two torsion pairs in $K(A-\mathcal{G P})$. This means $K(A-\mathcal{G P})$ has a $T T F$-triple $(K(A-\mathcal{P})$, $\left.\operatorname{Ker} R,(\operatorname{Ker} R)^{\perp}\right)$.

Acknowledgement The author would like to thank Professor Pu Zhang for his valuable comments on the work. 
Open Access This article is distributed under the terms of the Creative Commons Attribution License which permits any use, distribution, and reproduction in any medium, provided the original author(s) and the source are credited.

\section{References}

1. Avramov, L.L., Martsinkovsky, A.: Absolute, relative, and Tate cohomology of modules of finite Gorenstein dimension. Proc. Lond. Math. Soc. 85(3), 393-440 (2002)

2. Beilinson, A.A., Bernstein, J., Deligne, P.: Faisceaux pervers. In: Proceedings of the Conference Analysis and Topology on Singular Spaces, vol. 100. Luminy, 1981. Astérisque (1982)

3. Beligiannis, A.: Cohen-Macaulay modules, (co)torsion pairs and virtually Gorenstein algebras. J. Algebra 288, 137-211 (2005)

4. Beligiannis, A.: On algebras of finite Cohen-Macaulay type. Adv. Math. 226, 1973-2019 (2011)

5. Beligiannis, A., Reiten, I.: Homological and homotopical aspects of tosion theories. Mem. Amer. Math. Soc. 188, 1-207 (2007)

6. Bruns, W., Herzog, J.: Cohen-Macaulay Rings, revised edition. Cambridge Studies in Adv. Math., vol. 39. Cambridge Univ. Press (1998)

7. Chen, X.W.: Homotopy equivalences induced by balanced pairs. J. Algebra 324(10), 2718-2731 (2010)

8. Christensen, L.W., Frankild, A., Holm, H.: On Gorenstein projective, injective and flat dimensions-a functorial description with applications. J. Algebra 302(1), 231-279 (2006)

9. Enochs, E.E., Jenda, O.M.G.: Gorenstein injective and projective modules. Math. Z. 220(4), 611633 (1995)

10. Enochs, E.E., Jenda, O.M.G.: Relative homological algebra. De Gruyter Exp. Math., vol. 30. Walter De Gruyter Co. (2000)

11. Gao, N., Zhang, P.: Gorenstein derived categories. J. Algebra 323, 2041-2057 (2010)

12. Happel, D.: On Gorenstein algebras. Representation theory of finite groups and finitedimensional algebras. Prog. Math. 95, 389-404 (1991)

13. Holm, H., Jørgensen, P.: Compactly generated homotopy categories. Homology Homotopy Appl. 9(1), 257-274 (2007)

14. Holm, H.: Gorenstein homological dimensions. J. Pure Appl. Algebra 189(1-3), 167-193 (2004)

15. Jørgensen, P.: The homotopy category of complexes of projective modules. Adv. Math. 193, 223232 (2005)

16. Jørgensen, P.: Existence of Gorenstein projective resolutions and tate cohomology. J. Eur. Math. Soc. 9(1), 59-76 (2007)

17. Körrer, H.: Cohen-Macaulay modules on hpersurface singularities. Invent. Math. 88(1), 153-164 (1987)

18. Krause, H.: Smashing subcategories and the telescope conjecture-an algebraic approach. Invent. Math. 139, 99-133 (2000)

19. Li, Z.W., Zhang, P.: Gorenstein algebras of finite Cohen-Macaulay type. Adv. Math. 223(2), $728-734$ (2010)

20. Miyachi, J.: Localization of triangulated categories and derived categories. J. Algebra 141, 463483 (1991)

21. Neeman, A.: The Grothendieck duality theorem via Bousfield's techniques and Brown representability. J. Am. Math. Soc. 8(1), 205-236 (1996)

22. Neeman, A.: Triangulated categories. Annals of Mathematics Syudies, vol. 148, Princeton Univ. Press (2001) 